\title{
INCIDENTAL DETECTION OF PREVIOUSLY UNKNOWN BREAST CANCER ON Tc-99m MIBI SCINTIGRAPHY
}

\author{
Nur Gülce İşkan (D, Şeyma Gizem Orun² (D), Ezgi Gökdemir² (D), Gülay Durmuş Altun² (D)
}

${ }^{1}$ Trakya University School of Medicine, Edirne, TURKEY

${ }^{2}$ Department of Nuclear Medicine, Trakya University School of Medicine, Edirne, TURKEY

\begin{abstract}
Aims: Tc-99m methoxyisobutylisonitrile scintigraphy is a diagnostic method commonly used for cardiac perfusion imaging. It is also used for parathyroid, lung, breast, thyroid, brain, melanoma, lymphoma, bone, and soft tissue primary and secondary tumors imaging. Our case aims to report a breast cancer incidentally revealed by Tc- $99 \mathrm{~m}$ methoxyisobutylisonitrile scintigraphy. Case Report: A 49-year-old female patient was admitted to the cardiology department with atypical angina. Tc-99m methoxyisobutylisonitrile scintigraphy showed myocardial perfusion was within normal limits but a focal uptake was detected in the lateral superior quadrant of the left breast. Ultrasonography detected a lesion with irregular borders in the outer quadrant of the left breast and a lymph node with increased thickness of the cortex in the left axilla. Magnetic resonance imaging showed a mass with a spiculated contour in the outer quadrant of the left breast and lymph nodes with increased cortex thickness in both axillae. By the histopathologic examination, the specimen was diagnosed with invasive ductal carcinoma. Conclusion: Although Tc-99m methoxyisobutylisonitrile scintigraphy is mainly used for myocardial perfusion imaging, the entire image area should be examined in detail and further investigation should be done for incidental focal lesions that were previously undetected. Keywords: Tc-99m methoxyisobutylisonitrile, breast cancer, scintigraphy, incidental
\end{abstract}

\section{INTRODUCTION}

Tc-99m methoxyisobutylisonitrile (MIBI) is a lipophilic, monovalent cationic isonitrile compound. It passively diffuses across the cell membrane and is sequestered in the mitochondria (1). Tc99m MIBI scintigraphy is currently a commonly used diagnostic method for cardiac perfusion imaging (2). Myocardial perfusion scintigraphy is a noninvasive modality widely used in the evaluation of myocardial ischemia. Perfusion defects have been linked to areas supplied by significantly stenosed coronary arteries (3). It is also used for parathyroid imaging, especially for parathyroid adenomas and lung, breast, thyroid, brain, melanoma, lymphoma, bone, and soft tissue primary and secondary tumors imaging (4). With this case report, we aimed to report a breast cancer that was found incidentally by Tc-99m MIBI scintigraphy.

\section{CASE REPORT}

A 49-year-old female patient with rheumatic mitral valve disease and scoliosis was admitted to the cardiology department with atypical angina. After clinical examination, Tc-99m MIBI scintigraphy was requested for ischemia detection.

Myocardial perfusion was within normal limits in the MIBI images and ischemia was ruled out, however, a focal uptake was detected in the lateral superior quadrant of the left breast (Figure $1 \mathrm{~A}-\mathrm{B}$ ). Therefore, the patient was referred to the general surgery department for further examination. After clinical exami- nation, breast ultrasonography (USG) and breast magnetic resonance imaging (MRI) were requested. USG revealed a $17 \times 8 \mathrm{~mm}$ lymph node with increased cortex thickness in the left axillae.
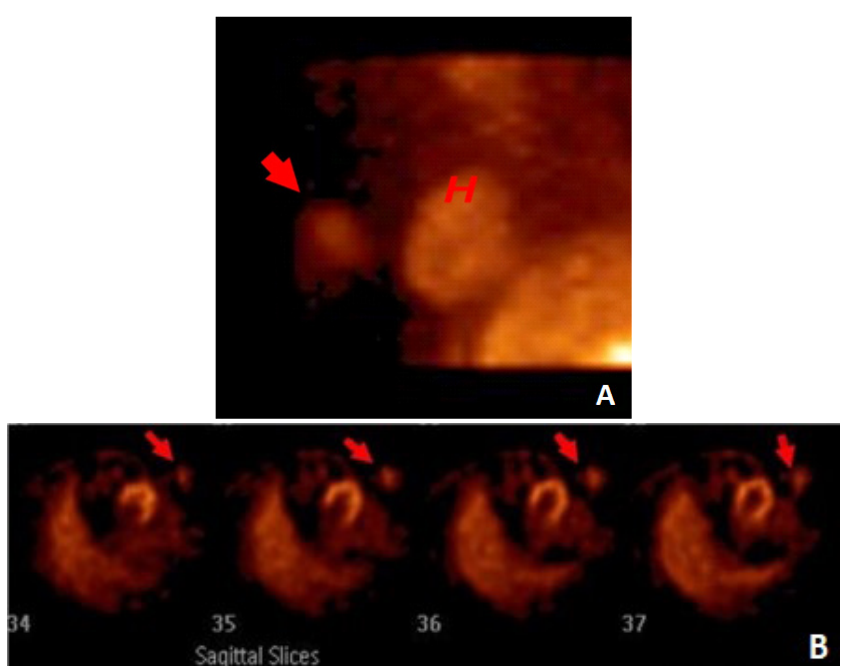

Figure 1: A: Tc-99m MIBI scintigraphy raw image indicating focal uptake in the lateral superior quadrant of the left breast $(H$ : heart) (Arrow). B: Tc-99m MIBI scintigraphy sagittal sectional images with focal uptake in the left breast (Arrows). 
In the outer quadrant of the left breast, a lesion with $35 \times 22 \mathrm{~mm}$ in size and irregular borders was observed. This lesion also created retraction in surrounding tissues. A few more satellite nodules were observed in the neighborhood of the lesion. The MRI scan detected an irregularly shaped lesion in the outer quadrant of the left breast, $36 \times 12 \mathrm{~mm}$ in size, with a contoured spicule that has been followed (Figure 2). The mass was found to be extended along the lesion ductus. The left breast skin seemed diffusely thickened. An approximately $10 \times 3 \mathrm{~mm}$ asymmetric contrast enhancement area is observed in the outer quadrant of the right breast areola plane. In both axillae, lymph nodes with increased cortex thickness were also seen.

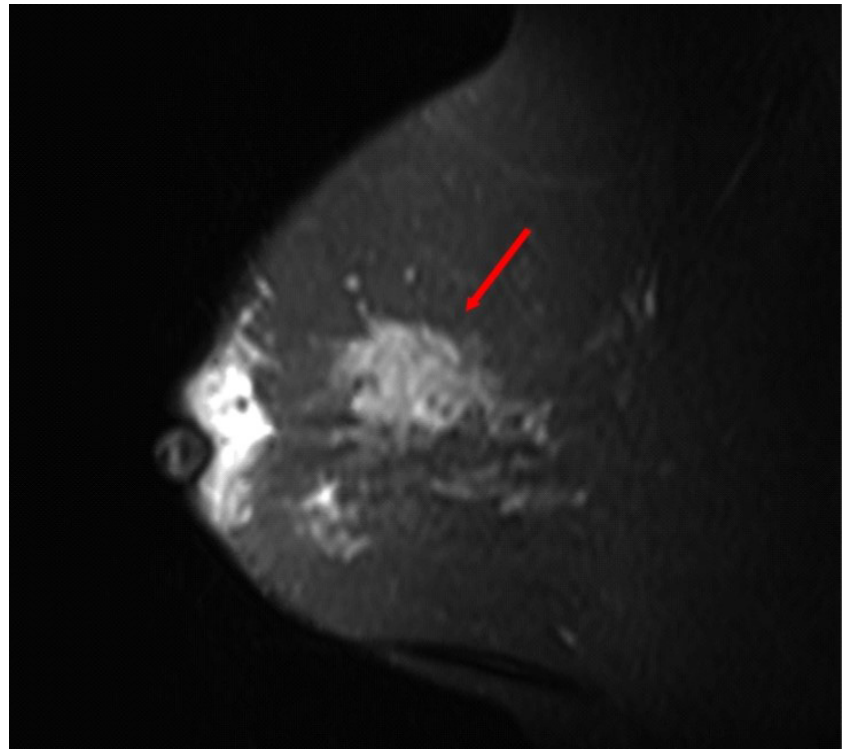

Figure 2: Irregularly shaped lesion (red arrow) in the outer quadrant of the left breast on MRI.

Histopathological evaluation of the surgical specimen confirmed the incidental diagnosis of breast cancer. The histopathologic examination result of the specimen was invasive ductal carcinoma. The tumor was staged as pT1N1M0.

\section{DISCUSSION}

Tc-99m MIBI is especially used for myocardial perfusion imaging. However, incidental uptake on the areas of rotating raw SPECT images have also been detected (2). These findings may alert physicians to a condition that needs to be further examined, such as breast cancer.

In tumor cells, MIBI retention is dependent to cellular and mitochondrial membrane potential and ATP-powered efflux pump such as P-glycoprotein (5). The initial use of Tc-99m MIBI for breast cancer was reported by Aktolun et al. (6) in 1992. Afterwards, it has been evaluated by many other researchers for the imaging of breast cancer lesions and the prognostic value for chemotherapy response (2). It was found to make unique contributions in select patients such as women with dense breasts or nonpalpable mammographically indeterminate lesions or palpable abnormalities (7).
Although scintimammography is not used as the primary diagnostic method, it can be used in select cases where other diagnostic methods are indeterminate. It is also recommended for sentinel lymph node detection in breast cancer $(8,9)$. The sensitivity and specificity of Tc-99m MIBI scintigraphy are $75.4 \%$ and $82.7 \%$, respectively (7). Therefore, it is important to evaluate the chest wall, even if the examination was performed for other purposes such as for myocardial perfusion, as seen in this case.

In imaging studies, incidental lesions are not uncommon. There are cases reported in the literature in which breast cancer was incidentally detected during imaging with Tc-99m MIBI for the diagnosis of other pathologies $(10,11)$. As in this case, patients may have breast lesions or other masses that are not yet known by their clinicians.

In conclusion, even though Tc-99m MIBI scintigraphy is mainly used for myocardial perfusion imaging, the entire image field should be evaluated meticulously, and any incidental focal lesions should be further examined in case of a previously undetected condition.

\section{Ethics Committee Approval: N/A}

Informed Consent: Written informed consent was obtained from the patient.

Conflict of Interest: The authors declared no conflict of interest.

Author Contributions: Concept: ŞGO, NGİ, EG. Design: ŞGO, NGİ, EG. Supervision: GDA. Resources: GDA. Materials: GDA, NGİ, EG, ŞGO. Data collection and/or processing: ŞGO, EG. Analysis and/or Interpretation: GDA. Literature Search: NGİ, EG, ŞGO. Writing Manuscript: NGİ, EG, ŞGO, GDA. Critical Review: GDA, NGİ, ŞGO, EG.

Financial Disclosure: The authors declared that this study received no financial support.

\section{REFERENCES}

1. Palestro CJ, Tomas MB, Tronco GG. Radionuclide imaging of the parathyroid glands. Sem Nucl Med 2005;6(1):266-76.

2. Muzaffar R, Raslan O, Ahmed F et al. Incidental findings on myocardial perfusion SPECT images. J Nucl Med Technol 2017;45:175-80.

3. Fathala A. Myocardial perfusion scintigraphy: Techniques, interpretation, indications and reporting. Ann Saudi Med 2011;31(6):625-34.

4. Bauer JL, Toluie S, Thompson LDR. Metastases to the parathyroid glands: A comprehensive literature review of 127 reported cases. Head Neck Pathol 2018;12(4):534-41.

5. Yoon HJ, Kim Y, Lee JE et al. Background 99mTc-methoxyisobutylisonitrile uptake of breast-specific gamma imaging in relation to background parenchymal enhancement in magnetic resonance imaging. Eur Radiol 2015;25:32-40.

6. Aktolun C, Bayhan H, Kir M. Clinical experience with Tc-99m MIBI imaging in patients with malignant tumors preliminary results and comparison with TI-201. Clin Nucl Med 1992;17(3):171-6.

7. Khalkhali I, Villanueva-Meyer J, Edell SL et al. Diagnostic accuracy of $99 \mathrm{mTc}$-sestamibi breast imaging: Multicenter trial results. J Nucl Med 2000;41:1973-9.

8. Mathieu I, Mazy S, Willemart B et al. Inconclusive triple diagnosis in breast cancer imaging: Is there a place or scintimammography? J Nucl Med 2005;46:1574-81.

9. Sadeghi R, Zakavi SR, Forghani MN et al. The efficacy of Tc-99m sestamibi for sentinel node mapping in breast carcinomas: A comparison with Tc-99m antimony sulphide colloid. Nucl Med Rev Cent East Eur 2010;13:1-4.

10. Seker D, Seker G, Öztürk E et al. An incidentally detected breast cancer on Tc99m MIBI cardiac scintigraphy. J Breast Cancer 2012;15(2):252-4.

11. Homma $\mathrm{T}$, Manabe $\mathrm{O}$, Ichinokawa $\mathrm{K}$ et al. Breast cancer detected as an incidental finding on 99mTc-MIBI scintigraphy. Acta Radiologica Open 2017;6(7):1-3. 